\section{Tropical Journal of Ophthalmology and Otolaryngology}

2020 Volume 5 Number 8 November-December

\title{
Randomized Controlled Trial of varied incision sites in Manual Small Incision Cataract Surgery done in a Medical College Hospital in Kancheepuram District, Tamil Nadu, India
}

\author{
Cornelius Gnanadurai J. ${ }^{1 *}$, Karunanidhi S. ${ }^{2}$ \\ DOI: https://doi.org/10.17511/jooo.2020.i08.07 \\ 1* J. Samuel Cornelius Gnanadurai, Professor, Department of Ophthalmology, SRM Medical College Hospital and Research Centre, Chennai, \\ Tamil Nadu, India. \\ 2 S. Vimala Karunanidhi, Senior Resident, Department of Ophthalmology, SRM Medical College Hospital and Research Centre, Chennai, \\ Tamil Nadu, India.
}

\begin{abstract}
Aim: To compare visual outcome, postoperative surgically induced astigmatism (SIA), and postoperative complications of superior and temporal scleral incision in manual small incision cataract surgery (MSICS). Materials and Methods: A Prospective Randomized, parallel-group, active-controlled Trial Study of 80 cases of senile/pre-senile cataract. 40 cases were randomly assigned (computer-generated randomization) to superior incision and 40 cases to the temporal incision. Results: The majority of patients (38 patients- $47.5 \%$ ) were in the age group of 56$65 y e a r s$. The next common age group was $66-75$ years(31 patients-38.75\%). In the superior MSICS group, the majority of patients (28 patients-70\%) had a moderate post-operative unaided visual acuity of $6 / 18-6 / 12$. In the temporal MSICS group, majority of patients(33patients-82.5\%) had a good post-operative unaided visual acuity of 6/9-6/6. The mean surgically induced astigmatism(SIA) in the superior MSICS group was 1.12 and in the temporal MSICS group was 0.45 with a difference of 0.67 . The SIA values were analyzed using the unpaired T-test which showed a value of 9.967 which was statistically significant( $P$ value of 0.0001$)$. Conclusion: This study has shown that the temporal approach for Manual small incision cataract surgery(MSICS) has less postoperative astigmatism and better postoperative unaided visual acuity compared to the superior approach.
\end{abstract}

Keywords: Manual small incision cataract surgery(MSICS), Surgically induced astigmatism(SIA), Superior scleral incision, Temporal scleral incision

Corresponding Author

J. Samuel Cornelius Gnanadurai, Professor, Department of Ophthalmology, SRM Medical College Hospital and Research Centre, Chennai, Tamil Nadu, India.

Email: samueldurai@yahoo.com
How to Cite this Article

To Browse

Gnanadurai J S C, Karunanidhi S V. Randomized Controlled Trial of varied incision sites in Manual Small Incision Cataract Surgery done in a Medical College Hospital in Kancheepuram District, Tamil Nadu, India. Trop J Ophthalmol Otolaryngol. 2020;5(8):237-242. Available From

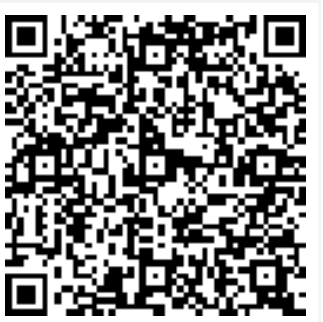

https://opthalmology.medresearch.in/index.php/jooo /article/view/178

Manuscript Received 2020-12-10

Conflict of Interest No
Review Round 1 2020-12-20

Funding Nil

Review Round 2
2020-12-25
Ethical Approval
Yes

Review Round 3

Accepted 2020-12-28

Plagiarism $\mathbf{X}$-checker $8 \%$

(c) 2020 by J. Samuel Cornelius Gnanadurai, S. Vimala Karunanidhiand Published by Siddharth Health Research and Social Welfare Society. This is an Open Access article licensed under a Creative Commons Attribution 4.0 International License https://creativecommons.org/licenses/by/4.0/ unported [CC BY 4.0]. 


\section{Introduction}

Senile Cataract is the most important cause of reversible Blindness in India and surgery is the only available intervention. Phacoemulsification is considered the gold standard procedure for cataracts. However, Manual small incision cataract surgery (MSICS) has emerged as a popular technique [1] and it has been possible to deliver cost-effective quality surgery to the masses in developing countries, especially among the rural and suburban population. Post-operative astigmatism has remained an obstacle to the achievement of good uncorrected visual acuity after cataract surgery. Recent progress in cataract surgery has heightened patient's expectations of the outcome. Good postoperative vision without spectacles is considered the norm. Thus, control of postoperative astigmatism [2] is the key to meeting these expectations. With the advent of sutureless small incision cataract surgery (MSICS), the amount of surgically induced astigmatism (SIA) has significantly reduced.

A variety of scleral incisions are being used in manual SICS such as superior, temporal, and superotemporal [3] to keep postoperative astigmatism to a minimum. All scleral pocket incisions share the advantages of intra and postoperative wound stability which include early healing, faster visual recovery, and superior astigmatism control. Site and size of scleral incisions are the factors that influence surgically induced astigmatism (SIA). The larger the incision size, the more is astigmatism. However, when the size is kept constant, the main determinant of surgically induced astigmatism(SIA) is the site of scleral incision [3]. The superior scleral incision is commonly performed as it is easy and has forehead support of the patients but it is said to cause more surgically induced astigmatism (SIA). The temporal scleral incision is difficult to make due to lack of support and less area of surgical exposure, but it is supposed to result in less surgically induced astigmatism (SIA). Another factor to be taken into consideration is the safety and incidence of postoperative complications with both these scleral incisions. The superior scleral incision is covered by the upper lid, and therefore the incidence of postoperative infection is supposed to be less with the superior incision. The temporal scleral incision is more exposed, and the chances of postoperative infection are supposed to be more with the temporal incision.
The current study was done to compare the surgically induced astigmatism and postoperative vision and complications of two groups of manual SICS done through superior and temporal scleral incisions.

\section{Materials and Methods}

Aim of the study: To compare visual outcome, postoperative surgical induced astigmatism(SIA) and postoperative complications of superior and temporal scleral incision in manual small incision cataract surgery (MSICS).

Study Design: A Prospective Randomized, parallelgroup, active-controlled Trial Study of 80 cases of senile/pre-senile cataract. 40 cases were randomly assigned(computer-generated randomization) to superior incision and 40 cases to the temporal incision.

Sample size: 80 patients aged $>45$ years. Duration of Study: 6months(surgery/collection of data-3months and follow up period-3months).

Diagnostic Methods: Patients will be randomly assigned(computer-generated randomization) to either of the two groups, each group will have 40 patients.Group1 will undergo manual SICS with superior tunnel incision and Group2 will undergo manual SICS with temporal tunnel incision. All patients will undergo a complete ocular examination which includes visual acuity, slit-lamp biomicroscopy, tonometry, and fundus examination. Astigmatism is measured by an auto refractometer and Keratometer. IOL power is calculated with contact A-scan biometry using the SRK II formula.

\section{Inclusion criteria:}

01. Senile/presenile cataract

02 . Age $>45$ years.

03. Immature/mature cataract

\section{Exclusion criteria:}

01. Brown/hard cataract, traumatic cataract

02. Congenital cataract, complicated cataract

03. Corneal surface irregularities or opacities

04. Glaucoma/uveitis

05. >1.5 D of keratometric astigmatism

06. Previous intraocular surgery.

07. Diabetic retinopathy/ hypertensive retinopathy

08. Amblyopia 
Surgical procedure: All patients will be operated on under peribulbar anesthesia. Fornix based conjunctival flap peritomy is done. A $6.5 \mathrm{~mm}$ frown scleral incision, $1.5 \mathrm{~mm}$ posterior to limbus is made superiorly in group1 and temporally in group2 with Bard-Parker no15 blade. A crescent blade is used to dissect $1.5 \mathrm{~mm}$ into the clear cornea to form a tunnel. The side port is made and the anterior chamber is filled with the viscoelastic solution (Hydroxypropyl methylcellulose 2\%). Anterior capsulorhexis is performed with bent 26 gauge needle cystectomy. Entry into the anterior chamber is made with a sharp $3.2 \mathrm{~mm}$ keratome and extended sideways to create a self-sealing corneal valve. The internal opening is $1 \mathrm{~mm}$ more than the external opening to facilitate the delivery of the nucleus. Hydro-dissection is performed with ringer lactate solution. Hydro-dissection is continued until the upper pole of the nucleus prolapses out of the capsular bag. Through the sclera tunnel, irrigating wire Vectis is passed under the nucleus, and the nucleus is delivered by pulling the Vectis out slightly depressing the scleral side of the incision. The cortical matter is aspirated with a simcoe two-way irrigation and aspiration cannula. In the presence of a viscoelastic solution, a rigid $6 \mathrm{~mm}$ PMMA intraocular lens is implanted in the capsular bag. The viscoelastic solution is removed by irrigation and corneal stromal hydration is done at the wound edges. The eye is bandaged for 24 hours. Postoperative antibiotic and steroid eye drops are given.

Follow-up: All patients will be examined on Day 1, 1 week ( 7 days), 6 weeks ( 45 days), and 3 months (90 days). Uncorrected and best-corrected visual acuity, slit-lamp examination, auto refractometer, and keratometry examination will be done on all visits.

Statistical Analysis: The postoperative surgically induced astigmatism(SIA) is calculated by comparing pre and postoperative keratometric values as described by Holladay et al [4] using the SIA calculator version 2.1. The values are statistically analyzed using the unpaired T-test.

Intervention: This is a surgical intervention study. Manual SICS is a safe and effective procedure under expert hands with excellent intra and postoperative stability and very minimal complications.

Ethics: Clearance was obtained from the scientific and ethical committee of SRM Medical College Hospital and Research Centre for the study.
Written and informed consent was obtained from all patients in their local language. They were given an option to quit the study if desired by them. All data will be kept confidential.

\section{Results}

\section{Age Distribution}

A total of 80 patients took part in the study out of which 11 patients $(13.75 \%)$ were in the age group of 46-55 years. The majority of patients (38 patients- $47.5 \%$ ) were in the age group of $56-$ $65 y e a r s$. The next common age group was $66-75$ years (31 patients-38.75\%).

Table-1: Age distribution.

\begin{tabular}{|l|l|l|}
\hline \multicolumn{1}{|c|}{ Age group(years) } & \multicolumn{1}{|c|}{ Frequency } & \multicolumn{1}{c|}{ Frequency in \% } \\
\hline $46-55$ & 11 & 13.75 \\
\hline $56-65$ & 38 & 47.5 \\
\hline $66-75$ & 31 & 38.75 \\
\hline
\end{tabular}

Post-operative unaided visual acuity

Among the 40 patients in the superior MSICS group, 9 patients(22.5\%) had a poor post-operative unaided visual acuity of $6 / 36-6 / 24$ and 28 patients(70\%) had a moderate post-operative unaided visual acuity of $6 / 18-6 / 12$. Only 3patients $(7.5 \%)$ had a good post-operative unaided visual acuity of 6/9-6/6.

Among the 40 patients in the temporal MSICS group, 33 patients $(82.5 \%)$ had a good postoperative unaided visual acuity of $6 / 9-6 / 6$. Only 7 patients $(17.5 \%)$ had a moderate post-operative unaided visual acuity of $6 / 18-6 / 12$. None of the patients had poor post-operative unaided vision.

Table-2: Post-operative unaided visual acuity.

\begin{tabular}{|l|l|l|l|l|}
\hline \multicolumn{1}{|c|}{ Incision } & \multicolumn{1}{|c|}{$\mathbf{6 / 3 6 - 6 / 2 4}$} & \multicolumn{1}{|c|}{$\mathbf{1 8 - 6 / 1 2}$} & \multicolumn{1}{|c|}{$\mathbf{6 / 9 - 6 / 6}$} & Total \\
\hline Superior & $9(22.5 \%)$ & $28(70 \%)$ & $3(7.5 \%)$ & 40 \\
\hline Temporal & $0(0 \%)$ & $7(17.5 \%)$ & $33(82.5 \%)$ & 40 \\
\hline
\end{tabular}

\section{Surgically induced astigmatism (SIA)}

SIA calculator version 2.1 was used to calculate the surgically induced astigmatism (SIA) in the superior incision and temporal incision of Manual small incision cataract surgery(MSICS). The mean surgically induced astigmatism (SIA) in the superior MSICS group was 1.12 and in the temporal MSICS group was 0.45 with a difference of 0.67 . The SIA values were analyzed using the unpaired T-test which showed a value of 9.967 which was statistically significant (P-value of 0.0001). 
In both groups, there was no sign of any postoperative infections. Apart from minimal corneal edema and minimal anterior chamber reaction which cleared with topical medications, there were no other significant postoperative complications noted in both the groups.

Table-3: Surgically induced astigmatism(SIA).

\begin{tabular}{|l|l|l|l|l|}
\hline Incision & \multicolumn{1}{|c|}{ Mean SIA } & SEM & Unpaired T-test & P-value \\
\hline Superior & 1.12 & 0.06 & 9.967 & 0.0001 \\
\hline Temporal & 0.45 & 0.03 & & \\
\hline
\end{tabular}

\section{Discussion}

Though phacoemulsification is technically superior and the gold standard in cataract surgery, it is not always preferred due to its high cost and not suitable for very dense cataracts. Manual small incision cataract surgery (MSICS) is the preferred alternative to phacoemulsification because of its lower cost, safety, and easy learning curve [5]. Manual small incision cataract surgery (MSICS) is the preferred cataract surgery for a developing country. The visual outcome in Manual small incision cataract surgery (MSICS) is equivalent to phacoemulsification. In very dense cataracts, Manual small incision cataract surgery (MSICS) is preferred over phacoemulsification. The main disadvantage of Manual small incision cataract surgery (MSICS) is the higher surgically induced astigmatism because of larger incision compared to phacoemulsification. Higher postoperative astigmatism is an important cause of poor uncorrected visual acuity following cataract surgery. By trying to change the type of surgical incision(straight, smile, frown incision), site of incision(superior, superotemporal, temporal) and size of incision( $6-7 \mathrm{~mm})$, the postoperative uncorrected visual acuity and lessen the postoperative surgical induced astigmatism(SIA) can be improved. Therefore, a variety of scleral incisions have been tried in Manual small incision cataract surgery (MSICS) such as superior, superotemporal, and temporal incisions to reduce postoperative astigmatism. For developing countries like India, manual SICS was affordable and had encouraging results [6]. Since then various modifications have been tried. It has been seen that frown incision offers minimal astigmatism in SICS [7]. But uncorrected visual acuity was decreased mostly due to postoperative astigmatism [8]. Malik VK et al compared 110 eyes of manual SICS of which 54 eyes underwent superior incision and 54 eyes underwent temporal incision [9].
He concluded that SICS with the temporal approach provides a better stabilization of the refraction with a significantly less surgically induced astigmatism(SIA) than the superior approach. Gokhale et al compared 45 eyes, 15 eyes each undergoing superior, superotemporal, and temporal scleral incisions [10]. The study found that induced astigmatism was lower in the temporal and superotemporal groups compared to the superior group. Ganagi et al compared 88 patients of which 44 underwent superior straight incision and 44 underwent temporal straight incision [11]. Postoperative astigmatism and better visual acuity were seen in those with a temporal straight incision as compared to a superior straight incision. Magdum et al conducted a prospective randomized comparative study of 100 cases of senile or presenile cataract [12]. 50 cases received superior scleral incision and 50 cases received temporal scleral incision. This study revealed that the temporal approach produces less postoperative astigmatism and has manifold advantages over superior incision with excellent visual outcomes.

Patients with senile/pre-senile cataract aged above 45 years were included in the present study. A total of 80 patients took part in the study out of which 11 patients $(13.75 \%)$ were in the age group of $46-55$ years. The majority of patients (38 patients$47.5 \%)$ were in the age group of $56-65$ years. The next common age group was $66-75$ years (31 patients-38.75\%). Among the 40 patients in the superior MSICS group, 9 patients $(22.5 \%)$ had a poor post-operative unaided visual acuity of $6 / 36$ $6 / 24$ and 28 patients (70\%) had a moderate postoperative unaided visual acuity of $6 / 18-6 / 12$. Only 3 patients $(7.5 \%)$ had a good post-operative unaided visual acuity of $6 / 9-6 / 6$. Among the 40 patients in the temporal MSICS group, 33 patients (82.5\%) had a good post-operative unaided visual acuity of 6/9$6 / 6$. Only 7 patients $(17.5 \%)$ had a moderate postoperative unaided visual acuity of 6/18-6/12. None of the patients had poor post-operative unaided vision. SIA calculator version 2.1 was used to calculate the surgically induced astigmatism (SIA) in the superior incision and temporal incision of Manual small incision cataract surgery (MSICS). The mean surgically induced astigmatism(SIA) in the superior MSICS group was 1.12 and in the temporal MSICS group was 0.45 with a difference of 0.67 . The SIA values were analyzed using the unpaired T-test which showed a value of 9.967 which was statistically significant( $P$-value of 0.0001$)$. 
The temporal approach for Manual small incision cataract surgery (MSICS) has yielded excellent results as it induces with the rule astigmatism (WTR). This counteracts the preoperative against the rule astigmatism (ATR) which is more common in the elderly and thereby provides a better postoperative uncorrected visual acuity [13]. The temporal incision is further away from the visual axis compared to the superior incision and therefore any flattening due to the surgical incision is less likely to affect the corneal curvature. In the case of superior incision, the disadvantage is that eyelid blinking, as well as gravity, tends to have a drag on the surgical incision. The superior approach for manual small incision cataract surgery (MSICS) induces against the rule astigmatism (ATR) which can aggravate the preoperative existing against the rule astigmatism (ATR) which is common in the elderly, thereby reducing the postoperative uncorrected visual acuity.

Some studies have shown a higher incidence of bacterial endophthalmitis associated with temporal scleral incision [14]. However, in the present study, there was no sign of any postoperative infections in both groups. Apart from minimal corneal edema and minimal anterior chamber reaction which cleared with topical medications, there were no other significant postoperative complications noted in both the groups.

The limitation of the present study is the small sample size and limited follow-up. Also, the current study did not try to correct any pre-existing astigmatism which would have given additional information to the present study.

\section{Conclusion}

The temporal approach for Manual small incision cataract surgery(MSICS) has a better postoperative unaided visual acuity compared to the superior approach. In the temporal MSICS group, the majority of patients( 33patients-82.5\%) had a good post-operative unaided visual acuity of 6/9-6/6, whereas in the superior MSICS group only 3 patients $(7.5 \%)$ had a good post-operative unaided visual acuity of $6 / 9-6 / 6$. In the superior MSICS group, the majority of patients(28patients-70\%) had a moderate post-operative unaided visual acuity of $6 / 18-6 / 12$.

The temporal approach for Manual small incision cataract surgery(MSICS) has less postoperative astigmatism compared to the superior approach.
The mean surgically induced astigmatism(SIA) in the superior MSICS group was 1.12 and in the temporal MSICS group was 0.45 with a difference of 0.67. A simple modification in incision placement from superior incision to temporal incision produces comparable results to other sophisticated procedures like phacoemulsification and hence offers a way to obtain better surgical and visual outcomes with limited resources.

Both the superior and temporal approach for Manual small incision cataract surgery(MSICS) were found to be very safe with no significant postoperative complications.

\section{What does the study add to the existing study?}

Manual small incision cataract surgery(MSICS) has emerged as a popular technique and it has been possible to deliver cost-effective quality surgery to the masses in developing countries, especially among the rural and suburban population. Postoperative astigmatism has remained an obstacle to the achievement of good uncorrected visual acuity after cataract surgery. The main determinant of surgically induced astigmatism (SIA) is the site of the scleral incision. This study has shown that the temporal approach for Manual small incision cataract surgery(MSICS) has less postoperative astigmatism and better postoperative unaided visual acuity compared to the superior approach. The temporal approach is safe with no significant postoperative complications. Even though superior incision remains the choice with many surgeons due to convenience and protective effect of the upper lid, and the temporal scleral incision is difficult to make due to lack of support and less area of surgical exposure, it is better to switch to temporal MSICS for the benefit of the patient.

\section{Author's contributions}

Dr. J. Samuel Cornelius Gnanadurai: Concept, study design, the definition of intellectual content, literature search, Scientific and ethical committee presentation, patient selection, clinical studies, data collection, data summarization and interpretation, Statistical analysis, manuscript preparation, manuscript editing, and manuscript review.

Dr. S. Vimala Karunanidhi: Scientific and ethical committee presentation, patient selection, clinical studies, and data collection. 


\section{Reference}

01. Neumann AC, McCarty GR, Sanders DR, Raanan MG. Small incisions to control astigmatism during cataract surgery. J Cataract Refract Surg. $1998 ; 15(1) 78-84$.

doi: $\quad 10.1016 / s 0886-3350(89) 80144-0 \quad$ [Crossref]

02. Burgansky Z, Isakov I, Avizemer $H$, Bartov E. Minimal astigmatism after sutureless planned extracapsular cataract extraction. J Cataract Refract Surg. 2002;28(3)499-503.

doi: $10.1016 / \mathrm{s} 0886-3350(01) 01263-9$ [Crossref]

03. Radwan AA. Comparing surgical induced astigmatism through change of incision site in manual small incision cataract surgery. J Clinic Experiment Ophthalmol. 2011;2(161)2.

[Crossref]

04. Holladay JT, Dudeja TR, Koch DD. Evaluating and reporting astigmatism for individual and aggregate data. J Cataract Refract Surg. 1998;24(1)57-65.

doi: $10.1016 / s 0886-3350(98) 80075-8$ [Crossref]

05. Venkatesh $R$, Das $M$, Prashanth $S$, Muralikrishnan R. Manual small incision cataract surgery in eyes with white cataracts. Indian J Ophthalmol. 2005;53(3)173-176.

doi: $10.4103 / 0301-4738.16675$ [Crossref]

06. Gogate P, Deshpande M, Nirmalan PK. Why do phacoemulsification? Manual small-incision cataract surgery is almost as effective, but less expensive. Ophthalmol. 2007;114(5)965-968.

doi: $10.1016 / j . o p h t h a .2006 .08 .057$ [Crossref]

07. Singer JA. Frown incision for minimizing induced astigmatism after small incision cataract surgery with rigid optic intraocular lens implantation. J Cataract Refract Surg. 1991;17;677-688.

doi: $10.1016 / s 0886-3350(13) 80683-9$ [Crossref]

08. Yorston D, Gichuhi S, Wood M, Foster A. Does prospective monitoring improve cataract surgery outcomes in Africa?. Brit J Ophthalmol. 2002;86(5)543-547.

doi: $10.1136 /$ bjo.86.5.543 [Crossref]
09. Malik VK, Kumar S, Kamboj R, Jain C, Jain K. Comparison of astigmatism following manual small incision cataract surgery- superior versus temporal approach. Nepal J Ophthalmol. 2012;4(7)54-58.

doi: 10.3126/nepjoph.v4i1.5851 [Crossref]

10. Gokhale NS, Sawhney S. Reduction in astigmatism in manual small incision cataract surgery through change of incision site. Indian J Ophthal. 2005;53(3)201-203. doi: 10.4103/0301-4738.16684 [Crossref]

11. Ganagi SM, Bhaskar A, Gonsalves S. Comparison of postoperative astigmatism in superior versus temporal incision in manual small incision cataract surgery. IOSR-JDMS. 2016;15(12)32-34.

doi: $10.9790 / 0853-1512033234$ [Crossref]

12. Magdum RM, Gahlot A, Maheshgauri RD, Patel K. A comparative study of surgically induced astigmatism in superior and temporal scleral incisionin manual small incision cataract surgery. Nat J Med Res. 2012;2(4)497-500. [Crossref]

13. Reddy B, Raj A, Singh VP. Site of incision and corneal astigmatism in conventional SICS versus phacoemulsification. Ann Ophthalmol. 2007;39(3):209-216.

doi: 10.1007/s12009-007-0020-y [Crossref]

14. Lundstrom M, Wejde G, Stenevi U, Thorburn W, Montan P. Endophthalmitis after cataract surgery- A nationwide prospective study evaluating incidence in relation to incision type and location. Ophthalmol. 2007;114(5)866-870. doi: $\quad 10.1016 /$ j.ophtha.2006.11.025 [Crossref] 